\title{
SCATTERING FOR THE YANG-MILLS EQUATIONS
}

\author{
JOHN C. BAEZ
}

\begin{abstract}
We construct wave and scattering operators for the Yang-Mills equations on Minkowski space, $\mathbf{M}_{0} \cong \mathbf{R}^{4}$. Sufficiently regular solutions of the Yang-Mills equations on $\mathbf{M}_{0}$ are known to extend uniquely to solutions of the corresponding equations on the universal cover of its conformal compactification, $\widetilde{\mathbf{M}} \cong \mathbf{R} \times S^{3}$. Moreover, the boundary of $\mathbf{M}_{0}$ as embedded in $\widetilde{\mathbf{M}}$ is the union of "lightcones at future and past infinity", $C_{ \pm}$. We construct wave operators $W_{ \pm}$as continuous maps from a space $\mathbf{X}$ of time-zero Cauchy data for the Yang-Mills equations to Hilbert spaces $\mathbf{H}\left(C_{ \pm}\right)$of Goursat data on $C_{ \pm}$. The scattering operator is then a homeomorphism $S: \mathbf{X} \rightarrow \mathbf{X}$ such that $U W_{+}=W_{-} S$, where $U: \mathbf{H}\left(C_{+}\right) \rightarrow \mathbf{H}\left(C_{-}\right)$is the linear isomorphism arising from a conformal transformation of $\widetilde{\mathbf{M}}$ mapping $C_{-}$onto $C_{+}$. The maps $W_{ \pm}$and $S$ are shown to be smooth in a certain sense.
\end{abstract}

\section{INTRODUCTION}

The conformal invariance of the Yang-Mills equations in four dimensions greatly facilitates the study of the temporal asymptotic behavior of their solutions. There is a natural conformal embedding $l$ of Minkowski space, $\mathbf{M}_{0} \cong$ $\mathbf{R}^{4}$, into the universal cover of its conformal compactification, $\widetilde{\mathbf{M}} \cong \mathbf{R} \times S^{3}$. Conformally invariant wave equations on $\mathbf{M}_{0}$ may thereby be extended to corresponding equations on $\widetilde{\mathbf{M}}[1,2]$. In particular, if $A$ is a connection satisfying the Yang-Mills equations on $\widetilde{\mathbf{M}}, i^{*} A$ satisfies the Yang-Mills equations on $\mathbf{M}_{0}$. Techniques used to prove global existence for the Yang-Mills Cauchy problem on $\mathbf{M}_{0}$ extend to prove global existence on $\widetilde{\mathbf{M}}$, allowing the derivation of precise asymptotics for Yang-Mills fields on $\mathbf{M}_{0}[3,4]$. Here we construct wave and scattering operators for the Yang-Mills equations on $\mathbf{M}_{0}$ in terms of the embedding $\imath: \mathbf{M}_{0} \rightarrow \widetilde{\mathbf{M}}$.

The boundary of $\mathbf{M}_{0}$ in $\widetilde{\mathbf{M}}$ is the union of two characteristic cones $C_{ \pm}$, the "lightcones at past and future infinity". Points of $C_{ \pm}$correspond to limits of null lines in $\mathbf{M}_{0}$ as the time coordinate in $\mathbf{M}_{0}$ approaches $\pm \infty$. The scattering theory of conformally invariant wave equations on $\mathbf{M}_{0}$ is thus closely related to the Goursat problem on $\widetilde{\mathbf{M}}$, in which solutions are determined by data on a characteristic cone [5]. Moreover the surface where the time coordinate of $\mathbf{M}_{0}$

Received by the editors April 28, 1988.

1980 Mathematics Subject Classification (1985 Revision). Primary 81E13; Secondary 35Q20, $58 \mathrm{G} 16,81 \mathrm{D} 25$. 
is zero is just the Cauchy surface $\{0\} \times S^{3} \subset \widetilde{\mathbf{M}}$ with a single point removed. For the equation $\square f+\lambda f^{3}=0$ this allowed the formulation of wave operators $W_{ \pm}$ as nonlinear maps from a Hilbert space of Cauchy data at time zero to Hilbert spaces of Goursat data on the cones $C_{ \pm}$[6]. These maps can be correlated with the traditional wave operators mapping sufficiently regular solutions of $\square f+\lambda f^{3}=0$ to temporally asymptotic solutions of the free wave equation. Moreover, the maps $W_{ \pm}$are smooth with smooth inverses, so the scattering operator $W_{+}\left(W_{-}\right)^{-1}$ exists and is a diffeomorphism [7].

A difficulty in extending this approach to the Yang-Mills equations is that global existence has not been proven for finite-energy Cauchy data, but only for data having more derivatives. The techniques used in [6] to solve the Goursat problem and invert the operators $W_{ \pm}$rely heavily upon the fact that the spaces of Goursat data have energy-type norms. Thus while we construct wave operators for the Yang-Mills equations and show they are smooth in a certain sense, we do not prove them invertible.

Nonetheless, a scattering operator of a different sort can be constructed. The group $S O^{\sim}(2,4)$ of conformal transformations of $\widetilde{\mathbf{M}}$ has a unique central element $\zeta$ mapping $C_{-}$onto $C_{+}$, and the action of this element $\zeta$ on solutions of conformally invariant equations on $\widetilde{\mathbf{M}}$ corresponds to scattering [5]. We show that for the Yang-Mills equations this map is smooth, and describe its relationship to the wave operators.

\section{THE SCATTERING OPERATOR}

First we recall the basic global existence theorem of [4]. We shall identify the universal cover of conformally compactified Minkowski space, $\widetilde{\mathbf{M}}$, with $\mathbf{R} \times S^{3}$ given the metric $d t^{2}-d s^{2}$, where $d t^{2}$ and $d s^{2}$ are the standard Riemannian metrics on $\mathbf{R}$ and $S^{3}$, respectively. Let $\mathbf{g}$ be the Lie algebra of a compact Lie group. Given a smooth manifold $M$, possibly with boundary, let $\Omega^{p}(M, \mathbf{g})$ denote the $g$-valued differential $p$-forms over $M$. (We use this notation in informal contexts when no particular degree of differentiability need be specified.) Following the notation of [8], the Yang-Mills equations for $A \in \Omega^{1}(\widetilde{\mathbf{M}}, \mathbf{g})$ may be written as:

$$
F=d A+\frac{1}{2}[A, A] ; \quad d * F+[A, * F]=0 .
$$

In temporal gauge, the $d t$ component of $A$ is assumed to vanish, where $t$ is the $\mathbf{R}$-valued coordinate on $\widetilde{\mathbf{M}}$. We shall identify elements $A \in \Omega^{1}(\widetilde{\mathbf{M}}, \mathbf{g})$ with vanishing $d t$ component with functions $A: \mathbf{R} \rightarrow \Omega^{1}\left(S^{3}, \mathbf{g}\right)$. Let $d_{s}$ denote exterior differentiation of $\mathrm{g}$-valued forms on $S^{3}$, and let ${ }_{s}$ denote the Hodge *-operator on g-valued forms on $S^{3}$ with respect to the metric $d s^{2}$. Given $A, B \in \Omega^{1}\left(s^{3}, \mathbf{g}\right)$, we make the following definitions:

$$
\begin{gathered}
A \times B=*_{s}[A, B], \quad[A ; B]=*_{s}\left[A, *_{s} B\right], \\
\nabla \cdot A=*_{s} d_{s} *_{s} A, \quad \nabla \times A=*_{s} d_{s} A,
\end{gathered}
$$


and given $f \in \Omega^{0}\left(S^{3}, \mathbf{g}\right)$ we define $\nabla f$ to be $d_{s} f$. Note that if $\Delta$ denotes the Laplace-Beltrami operator on $\Omega^{1}\left(S^{3}, \mathbf{g}\right)$, we have $\Delta A=\nabla \times(\nabla \times A)-\nabla(\nabla \cdot A)$. Using ' to denote $\partial_{t}$, and working in temporal gauge, the equations (1) are equivalent to the evolution equation

$$
A^{\prime \prime}+\nabla \times(\nabla \times A)+A \times(\nabla \times A)+\frac{1}{2} \nabla \times(A \times A)+\frac{1}{2} A \times(A \times A)=0
$$

together with the constraint

$$
\nabla \cdot A^{\prime}+\left[A ; A^{\prime}\right]=0 .
$$

Identifying $S^{3}$ with $S U(2)$, let $V_{i}, 1 \leq i \leq 3$, be an orthonormal basis of left-invariant vector fields on $S^{3}$. Let $\Omega_{k}^{1}\left(S^{3}, \mathbf{g}\right)$ denote the space of $\mathbf{g}$-valued one-forms on $S^{3}$ with all components lying in the Sobolev space $H^{k}\left(S^{3}\right)$, given the structure of a real Hilbert space with the following norm:

$$
\|A\|_{k}^{2}=\sum_{1 \leq i \leq 3} \int_{S^{3}}\left|(\Delta+1)^{k / 2} A\left(V_{i}\right)\right|^{2}
$$

where here $\Delta$ denotes the Laplacian as a selfadjoint operator on $L^{2}\left(S^{3}, \mathbf{g}\right)$, and $|\cdot|$ denotes any Hilbert norm on $\mathbf{g}$.

The evolution equation (2) may be desingularized by differentiating it with respect to $t$ and using the constraint (3) to rewrite the resulting term $\nabla \times\left(\nabla \times A^{\prime}\right)$ as $\Delta A^{\prime}-\nabla\left[A ; A^{\prime}\right]$. Let $\mathbf{H}$ denote the real Hilbert space $\Omega_{3}^{1}\left(S^{3}, \mathbf{g}\right) \oplus \Omega_{2}^{1}\left(S^{3}, \mathbf{g}\right) \oplus$ $\Omega_{1}^{1}\left(S^{3}, \mathbf{g}\right) ; \mathbf{H}$ will be used as a space of Cauchy data $\left(A, A^{\prime}, A^{\prime \prime}\right)$. Let $\mathbf{X}$ denote the set of Cauchy data in $\mathbf{H}$ satisfying (2) and (3), that is, those $\left(A_{1}, A_{2}, A_{3}\right) \in$ $\mathbf{H}$ such that $\nabla \cdot A_{2}+\left[A_{1} ; A_{2}\right]=0$ and

$$
A_{3}+\nabla \times\left(\nabla \times A_{1}\right)+A_{1} \times\left(\nabla \times A_{1}\right)+\frac{1}{2} \nabla \times\left(A_{1} \times A_{1}\right)+\frac{1}{2} A_{1} \times\left(A_{1} \times A_{1}\right)=0 .
$$

Note that $\mathbf{X}$ is a closed subset of $\mathbf{H}$; we give $\mathbf{X}$ the metric arising from the norm on $\mathbf{H}$. Let $L$ be the unbounded operator on $\mathbf{H}$ with domain $\Omega_{4}^{1}\left(S^{3}, \mathbf{g}\right) \oplus$ $\Omega_{3}^{1}\left(S^{3}, \mathbf{g}\right) \oplus \Omega_{2}^{1}\left(S^{3}, \mathbf{g}\right)$ given by

$$
L\left(A_{1}, A_{2}, A_{3}\right)=\left(A_{2}, A_{3},-(\Delta+1) A_{2}\right),
$$

and let $N: \mathbf{H} \rightarrow \mathbf{H}$ be the function given by

$$
N\left(A_{1}, A_{2}, A_{3}\right)=\left(0,0, k\left(A_{1}, A_{2}\right)\right)
$$

where

$$
\begin{gathered}
k\left(A_{1}, A_{2}\right)=\nabla\left[A_{1} ; A_{2}\right]-A_{1} \times\left(\nabla \times A_{2}\right)-A_{2} \times\left(\nabla \times A_{1}\right)-\nabla \times\left(A_{1} \times A_{2}\right) \\
-A_{1} \times\left(A_{1} \times A_{2}\right)-\frac{1}{2} A_{2} \times\left(A_{1} \times A_{1}\right)+A_{2} .
\end{gathered}
$$

It follows from results in [4] that $L$ generates a strongly continuous oneparameter semigroup on $\mathbf{H}$, and that the function $N$ is $C^{\infty}$ from $\mathbf{H}$ to $\mathbf{H}$ with all derivatives bounded on bounded sets.

The global existence result may be stated as follows: 
Lemma 1. For any Cauchy datum $u_{0} \in \mathbf{X}$ there is a unique continuous $u: \mathbf{R} \rightarrow \mathbf{X}$ satisfying

$$
u(t)=e^{L t} u_{0}+\int_{0}^{t} e^{L(t-s)} N(u(s)) d s
$$

Let $\left(A_{1}(t), A_{2}(t), A_{3}(t)\right)=u(t)$, and let $A \in \Omega^{1}(\widetilde{\mathbf{M}}, \mathbf{g})$ denote the $\mathbf{g}$-valued one-form corresponding to the continuous function $A_{1}: \mathbf{R} \rightarrow \Omega_{3}^{1}\left(S^{3}, \mathbf{g}\right)$. Then $A$ satisfies (2) and (3) in the distributional sense.

Proof. This follows from Theorems 3 and 4 of [4].

Define the map $T: \mathbf{R} \times \mathbf{X} \rightarrow \mathbf{X}$ as follows: given $u_{0} \in \mathbf{X}$, let $u: \mathbf{R} \rightarrow \mathbf{X}$ be the unique continuous solution of (4), and let $T(t) u_{0}=u(t) . T$ is clearly a group action of $\mathbf{R}$ on the space $\mathbf{X}$. This group action extends to an action of $\mathbf{R} \times S O(4)$, which is the identity component of the group of isometries of $\widetilde{\mathbf{M}}$ :

Lemma 2. The group $S O(4)$ has a strongly continuous orthogonal representation on $\mathbf{H}$ given by:

$$
g\left(A_{1}, A_{2}, A_{3}\right)=\left(\left(g^{-1}\right)^{*} A_{1},\left(g^{-1}\right)^{*} A_{2},\left(g^{-1}\right)^{*} A_{3}\right)
$$

where $g^{*}: \Omega^{1}\left(S^{3}, \mathbf{g}\right) \rightarrow \Omega^{1}\left(S^{3}, \mathbf{g}\right)$ denotes the pullback map induced by the diffeomorphism $g: S^{3} \rightarrow S^{3}$ corresponding to $g \in S O(4)$. If $g \in S O(4)$ and $u \in \mathbf{X}$, then $g u \in \mathbf{X}$. Moreover, there is a group action $\Gamma: \mathbf{R} \times S O(4) \mathbf{X} \rightarrow \mathbf{X}$ given by:

$$
\Gamma(t, g) u=T(t)(g u), \quad(t, g) \in \mathbf{R} \times S O(4), u \in \mathbf{H} .
$$

Proof. Since the action of $S O(4)$ on $S^{3}$ is isometric the linear transformation of $\mathbf{H}$ given by $u \mapsto g u$ is an isometry. The action of $S O(4)$ on any Sobolev space $H^{k}\left(S^{3}\right)$ is strongly continuous, so $(g, u) \mapsto g u$ is a continuous orthogonal representation. Moreover, if $u=\left(A_{1}, A_{2}, A_{3}\right) \in \mathbf{X}$ and $g u=\left(B_{1}, B_{2}, B_{3}\right)$, then simple computations show that $\nabla \cdot B_{2}+\left[B_{1} ; B_{2}\right]=0$ and

$$
B_{3}+\nabla \times\left(\nabla \times B_{1}\right)+B_{1} \times\left(\nabla \times B_{1}\right)+\frac{1}{2} \nabla \times\left(B_{1} \times B_{1}\right)+\frac{1}{2} B_{1} \times\left(B_{1} \times B_{1}\right)=0,
$$

hence $g u \in \mathbf{X}$. That $\Gamma$ is a group action follows from the fact that $T(t) g u=$ $g T(t) u$, a consequence of the $S O(4)$-invariance of equation (4).

As mentioned above, the action of the central element $\zeta \in S O^{\sim}(2,4)$ on the space of solutions of the Yang-Mills equation may be interpreted as the scattering operator [5]. In its action as a conformal transformation of $\widetilde{\mathbf{M}}$ it corresponds to the isometry $(\pi,-I) \in \mathbf{R} \times S O(4)$. Thus we define the scattering operator $S: \mathbf{X} \rightarrow \mathbf{X}$ by $S=\Gamma(\pi,-I)$. In a certain sense $S$ is smooth, but since $\mathbf{X}$ is not a submanifold of $\mathbf{H}$ [9], some care is required to make this precise. We will show that the action $\Gamma$ of $\mathbf{R} \times S O(4)$ on $\mathbf{X}$ extends to a local action $\bar{\Gamma}$ of $\mathbf{R} \times S O(4)$ as $C^{\infty}$ maps defined on neighborhoods of $\mathbf{X}$. 
More precisely:

Theorem 3. For any $(t, g) \in \mathbf{R} \times S O(4)$ there is an open set $U_{t} \subseteq \mathbf{H}$ containing $\mathbf{X}$ and a function $\bar{\Gamma}(t, g): U_{t} \rightarrow \mathbf{H}$ such that:

(a) The restriction of $\bar{\Gamma}(t, g)$ to $\mathbf{X}$ is $\Gamma(t, g)$.

(b) When both sides are defined, $\bar{\Gamma}(t, g) \bar{\Gamma}(s, h) v=\bar{\Gamma}(t+s, g h) v$.

(c) If $|s| \leq|t|$ then $U_{t} \subseteq U_{s}$. If $t \geq 0$ and $v \in U_{t}, \bar{\Gamma}(s, I) v$ is continuous as a function of $s$ from $[-t, t]$ to $\mathbf{H}$.

(d) For all $n \geq 1$ the function $\bar{\Gamma}(t, g): U_{t} \rightarrow \mathbf{H}$ has a continuous $n$th Fréchet derivative $D^{n} \bar{\Gamma}(t, g): \mathbf{H} \times \mathbf{H}^{n} \rightarrow \mathbf{H}$, where $\mathbf{H}^{n}$ denotes the $n$-fold product of $\mathbf{H}$. For all $v \in U_{t}$ the multilinear map $D^{n} \bar{\Gamma}(s, g) v: \mathbf{H}^{n} \rightarrow \mathbf{H}$ satisfies

$$
\sup \left\{\left\|D^{n} \bar{\Gamma}(s, g) v\right\|: g \in S O(4),|s| \leq|t|\right\}<\infty .
$$

Proof. Suppose that $u_{0} \in \mathbf{X}$ and $t \geq 0$. We shall show that for some $\varepsilon, M>0$, if $\left\|v_{0}-u_{0}\right\|<\varepsilon, 0 \leq t_{0} \leq t$, and $v:\left[-t_{0}, t_{0}\right] \rightarrow \mathbf{H}$ is a continuous functions such that

$$
v(s)=e^{L s} v_{0}+\int_{0}^{s} e^{L\left(s-s^{\prime}\right)} N\left(v\left(s^{\prime}\right)\right) d s^{\prime},
$$

then $\sup _{s \in\left[-t_{0}, t_{0}\right]}\|v(s)\| \leq M$. It then follows from the theory of nonlinear semigroups [10] that for any $v_{0} \in \mathbf{H}$ with $\left\|v_{0}-u_{0}\right\|<\varepsilon$ there is a unique continuous function $v:[-t, t] \rightarrow \mathbf{H}$ satisfying (5).

Let $F: \mathbf{R}^{2} \rightarrow \mathbf{R}$ be a continuous function, increasing in each variable, such that

$$
\|N(x)-N(y)\| \leq F(\|x\|,\|y\|)\|x-y\|, \quad x, y \in \mathbf{H} .
$$

Let $u: \mathbf{R} \rightarrow \mathbf{X}$ be as in Lemma 1 , and let

$$
M=\sup _{s \in[-t, t]}\|u(s)\|+1 \text { and } C=\sup _{s \in[-t, t]}\left\|e^{L s}\right\| .
$$

Choose $\varepsilon>0$ such that $\varepsilon C e^{C F(M, M) t}<1$. The value of $\varepsilon$ depends on $u_{0}$ and $t$; let us write it as $\varepsilon\left(u_{0}, t\right)$. By the definition of $\varepsilon$ and the $S O(4)$ invariance of the equations (4) and (5), we may choose this function so that $\varepsilon\left(u_{0}, t\right)=\varepsilon\left(g u_{0}, t\right)$ for all $g \in S O(4)$. Moreover we may choose this function so that $0 \leq s \leq t$ implies $\varepsilon\left(u_{0}, t\right) \leq \varepsilon\left(u_{0}, s\right)$.

Let $v_{0} \in \mathbf{H}$ have $\left\|v_{0}-u_{0}\right\|<\varepsilon\left(u_{0}, t\right)$. Let $0 \leq t_{0} \leq t$, and let $v:\left[-t_{0}, t_{0}\right] \rightarrow$ $\mathbf{H}$ be continuous, satisfying (5). The proof now proceeds by contradiction: assume that for some $s \in\left[-t_{0}, t_{0}\right]$ we have $\|v(s)-u(s)\|>1$. Let

$$
\tau=\inf \left\{|s|: s \in\left[-t_{0}, t_{0}\right],\|v(s)-u(s)\|>1\right\} \text {. }
$$

By continuity, either $\|v(\tau)-u(\tau)\|=1$ or $\|v(-\tau)-u(-\tau)\|=1$. We may assume that $\|v(\tau)-u(\tau)\|=1$, as the other case is analogous. By (4) and (5), for any $s \in[0, \tau]$ we have

$$
\begin{aligned}
\|v(s)-u(s)\| & \leq C \varepsilon\left(u_{0}, t\right)+C \int_{0}^{s} F\left(\left\|v\left(s^{\prime}\right)\right\|,\left\|u\left(s^{\prime}\right)\right\|\right)\left\|v\left(s^{\prime}\right)-u\left(s^{\prime}\right)\right\| d s^{\prime} \\
& \leq C \varepsilon\left(u_{0}, t\right)+C F(M, M) \int_{0}^{s}\left\|v\left(s^{\prime}\right)-u\left(s^{\prime}\right)\right\| d s^{\prime}
\end{aligned}
$$


Thus by Gronwall's inequality and our choice of $\varepsilon\left(u_{0}, t\right)$,

$$
\|v(\tau)-u(\tau)\| \leq \varepsilon\left(u_{0}, t\right) C e^{C F(M, M) \tau}<1,
$$

a contradiction. We conclude that for all $s \in\left[-t_{0}, t_{0}\right],\|v(s)-u(s)\| \leq 1$; hence $\sup _{s \in\left[-t_{0}, t_{0}\right]}\|v(s)\| \leq M$ as was to be shown.

Now for any $t \in \mathbf{R}$ define the open set $U_{t} \subseteq \mathbf{H}$ as follows:

$$
U_{t}=\bigcup_{u \in \mathbf{X}}\{v:\|v-u\| \leq \varepsilon(u,|t|)\} .
$$

Given $v_{0} \in U_{t}$, let $v:[-t, t] \rightarrow \mathbf{H}$ be the unique continuous function satisfying (5). Given $s$ with $|s| \leq t$ let $\bar{T}(s) v_{0}=v(s)$, and for $g \in S O(4)$ let

$$
\bar{\Gamma}(s, g) v_{0}=g \bar{T}(s) v_{0} \text {. }
$$

Statements (a) and (c) of the theorem now follow directly from the definitions. Statement (b) follows from the same arguments used in Lemma 2. To prove the differentiability claimed in (d) it suffices by (7) and Lemma 2 to prove the existence of continuous Fréchet derivatives $D^{n} \bar{T}(t): \mathbf{H} \times \mathbf{H}^{n} \rightarrow \mathbf{H}$. This follows by induction on $n$ using Theorem $\mathrm{B}^{\prime}$ of [11] and the fact that $N$ is $C^{\infty}$ with bounded derivatives on bounded sets. This theorem also establishes that for $v \in U_{t}$ and $|s| \leq|t|$, the Frechét derivative $D^{n} \bar{T}(s) v: \mathbf{H}^{n} \rightarrow \mathbf{H}$ is a strongly continuous function of $s$. By the uniform boundedness theorem we conclude that

$$
\sup _{|s| \leq|t|}\left\|D^{n} \bar{T}(s) v\right\|<\infty .
$$

Since $S O(4)$ acts as orthogonal transformations of $\mathbf{H}$ and $\bar{\Gamma}(s, g)=g \bar{T}(s)$, we have $\left\|D^{n} \bar{\Gamma}(s, g) v\right\|=\left\|D^{n} \bar{T}(s) v\right\|$, hence

$$
\sup \left\{\left\|D^{n} \bar{\Gamma}(s, g) v\right\|: g \in S O(4),|s| \leq|t|\right\}<\infty
$$

Corollary 4. For some open sets $U, V \subseteq \mathbf{H}$ containing $\mathbf{X}$, the scattering operator $S: \mathbf{X} \rightarrow \mathbf{X}$ extends to a diffeomorphism $\bar{S}: U \rightarrow V$.

Proof. Take $U=U_{\pi} \cap \bar{\Gamma}(-\pi,-I) U_{\pi}$, let $\bar{S}$ be the restriction of $\bar{\Gamma}(\pi,-I)$ to $U$, and let $V=\bar{S} U$. Then $\bar{S}: U \rightarrow V$ is $C^{\infty}$ by part (c) of the theorem, and if $v \in U, \bar{\Gamma}(-\pi,-I) \bar{S} v=v$ by part (b), so $\bar{\Gamma}(-\pi,-I) \mid V$ is a $C^{\infty}$ inverse of $\bar{S}$.

\section{THE WAVE OPERATORS}

In this section we describe wave operators mapping a Cauchy datum in $\mathbf{X}$ to restrictions of the corresponding solution of the Yang-Mills equation to the "lightcones at infinity", $C_{ \pm} \subset \widetilde{\mathbf{M}}$. Let $\rho$ denote the arclength from the point in $S^{3}$ corresponding to the identity of $S U(2)$. Let $C_{ \pm}$be the subsets of $\widetilde{\mathbf{M}}$ given by the equations $t= \pm(\pi-\rho)$. We define the Sobolev spaces $H^{k}\left(C_{ \pm}\right)$ through the identification of $C_{ \pm}$with $S^{3}$ by means of the one-to-one maps

$$
(t, x) \mapsto x, \quad(t, x) \in C_{ \pm} \subset \mathbf{R} \times S^{3} .
$$


The Sobolev space $H^{1}\left(C_{ \pm}\right)$is of particular significance, being the space of "finite-energy Goursat data" for the conformally invariant scalar wave equation on $\widetilde{\mathbf{M}}$, that is, the space of restrictions to $C_{ \pm}$of finite-energy solutions of $(\square+1) \varphi=0$, where $\square$ denotes the D'Alembertian on functions $\varphi: \widetilde{\mathbf{M}} \rightarrow \mathbf{R}$ [6]. Let $E_{ \pm}$denote the pullback of the bundle of g-valued one-forms on $\widetilde{\mathbf{M}}$ to the subspace $C_{ \pm}$, and let $\mathbf{H}\left(C_{ \pm}\right)$denote the space of sections of $E_{ \pm}$with all components lying in the Sobolev space $H^{1}\left(C_{ \pm}\right)$.

We shall formulate the wave operators for the Yang-Mills equations in terms of the spaces $\mathbf{H}\left(C_{ \pm}\right)$, as follows. As Cauchy datum $u \in \mathbf{X}$ determines a solution of the Yang-Mills equations in temporal gauge, $A \in \Omega^{1}(\widetilde{\mathbf{M}}, \mathbf{g})$. Letting $X_{i}$ denote the lifts to $\widetilde{\mathbf{M}}$ of the previously described vector fields $V_{i}$ on $S^{3}$, and letting $X_{0}=\partial_{t}$, the vector fields $X_{i}, 0 \leq i \leq 3$, form an orthonormal basis of vector fields on $\widetilde{\mathbf{M}}$. We shall show that the restrictions $A\left|C_{ \pm},\left(X_{i} A\right)\right| C_{ \pm}$, and $\left(X_{0} X_{i} A\right) \mid C_{ \pm}$are well-defined as elements of $\mathbf{H}\left(C_{ \pm}\right)$. Moreover, the functions $u \mapsto A\left|C_{ \pm}, u \mapsto\left(X_{i} A\right)\right| C_{ \pm}$, and $u \mapsto\left(X_{0} X_{i} A\right) \mid C_{ \pm}$extend to smooth functions defined on an open neighborhood of $\mathbf{X}$.

We make use of a lemma on the inhomogeneous wave equation for $\mathbf{g}$-valued one-forms. Let $R$ be the region of $\widetilde{\mathbf{M}}$ defined by $\{-\pi \leq t \leq \pi\}$, and let $\mathbf{V}$ be the space of $\mathrm{g}$-valued one-forms on $R$ with all components in the Sobolev space $L^{2}(R)$, given the structure of a real Hilbert space.

Lemma 5. If $A_{1}, A_{2} \in \Omega^{1}\left(S^{3}, \mathbf{g}\right)$ and $h \in \Omega^{1}(\widetilde{\mathbf{M}}, \mathbf{g})$ are $C^{\infty}$ sections, then there is a unique $C^{\infty}$ section $A \in \Omega^{1}(\widetilde{\mathbf{M}}, \mathbf{g})$ such that

$$
(\square+1) A=h,\left.\quad\left(A, X_{0} A\right)\right|_{t=0}=\left(A_{1}, A_{2}\right),
$$

where $\square$ denotes the D'Alembertian on $\Omega^{1}(\widetilde{\mathbf{M}}, \mathbf{g})$. The restrictions $A \mid C_{ \pm}$are functions of $h \mid R, A_{1}$, and $A_{2}$, and the functions $\left(h \mid R, A_{1}, A_{2}\right) \mapsto A \mid C_{ \pm}$thus defined extend uniquely to continuous maps

$$
T_{ \pm}: \mathbf{V} \oplus \Omega_{1}^{1}\left(S^{3}, \mathbf{g}\right) \oplus \Omega_{0}^{1}\left(S^{3}, \mathbf{g}\right) \rightarrow \mathbf{H}\left(C_{ \pm}\right) .
$$

Proof. This is a straightforward consequence of Lemma 6 of [7], which states an analogous result for the inhomogeneous scalar wave equation.

Note that by the theory of the Cauchy problem for the inhomogeneous wave equation, if $\left(A_{1}, A_{2}\right) \in \Omega_{1}^{1}\left(S^{3}, \mathbf{g}\right) \oplus \Omega_{0}^{1}\left(S^{3}, \mathbf{g}\right)$ and $h \in \mathbf{V}$ then there exists a unique $A \in \Omega_{1}^{1}(R, \mathbf{g})$ satisfying (6) in the distributional sense. The above lemma then allows us to define $A \mid C_{ \pm}$as an element of $\mathbf{H}\left(C_{ \pm}\right)$.

Theorem 6. Given $u \in \mathbf{X}$, let $\left(A_{1}(t), A_{2}(t), A_{3}(t)\right)=T(t) u$, and let $A$ denote the element of $\Omega^{1}(\widetilde{\mathbf{M}}, \mathbf{g})$ corresponding to $A_{1}: \mathbf{R} \rightarrow \Omega_{3}^{1}\left(S^{3}, \mathbf{g}\right)$. Then $A \mid C_{ \pm}$, $\left(X_{i} A\right) \mid C_{ \pm}$, and $\left(X_{0} X_{i} A\right) \mid C_{ \pm}$, where $0 \leq i \leq 3$, are well-defined elements of $\mathbf{H}\left(C_{ \pm}\right)$as in Lemma 5. Moreover, for some open set $U \subseteq \mathbf{H}$ containing $\mathbf{X}$ the functions $u \mapsto A\left|C_{ \pm}, u \mapsto\left(X_{i} A\right)\right| C_{ \pm}$, and $u \mapsto\left(X_{0}, X_{i} A\right) \mid C_{ \pm}$extend to $C^{\infty}$ maps from $U$ to $\mathbf{H}\left(C_{ \pm}\right)$. 
Proof. By symmetry it suffices to consider the restrictions to $C_{+}$. Suppose that $u \in U_{\pi}$, and for $|t| \leq \pi$ let $\left(A_{1}(t), A_{2}(t), A_{3}(t)=\bar{\Gamma}(t, I) u\right.$, where the open set $U_{\pi} \subseteq \mathbf{H}$ and $\bar{\Gamma}(t, I)$ are as in Theorem 3. Let $A$ denote the element of $\Omega^{1}(R, \mathbf{g})$ corresponding to $A_{1}:[-\pi, \pi] \rightarrow \Omega_{3}^{1}\left(S^{3}, \mathbf{g}\right)$, and let $h(u)$ denote the element of $\mathbf{V}$ corresponding to

$$
A_{3}+(\Delta+1) A_{1}:[-\pi, \pi] \rightarrow \Omega_{1}^{1}\left(S^{3}, \mathbf{g}\right) .
$$

By equation (5) and the definition of $L$ and $N$, we have $(\square+1) A=h(u)$ in the distributional sense. Note that by statements (c), (d) of Theorem 3 , the functions $u \mapsto h(u)$ is $C^{\infty}$ from $U_{\pi}$ to $\mathbf{V}$. Let us write $u=\left(B_{1}, B_{2}, B_{3}\right)$. By Lemma 5 and the remarks following, we may define $A \mid C_{+}=T_{+}\left(h(u), B_{1}, B_{2}\right)$, and conclude that $u \mapsto A \mid C_{+}$is a $C^{\infty}$ function from $U_{\pi}$ to $\mathbf{H}\left(C_{+}\right)$.

Next we treat $X_{i} A \mid C_{+}$. Since the Lie derivations $X_{i}$ commute with the differential operator $\square$, we have

$$
(\square+1) X_{i} A=X_{i} h(u)
$$

in the distributional sense. For $1 \leq i \leq 3$ the map $u \mapsto X_{i} h(u)$ is $C^{\infty}$ from $U_{\pi}$ to $\mathbf{V}$, by Theorem 3. Moreover, by (5) we have

$$
X_{0} h(u)=X_{0}\left(A_{3}+(\Delta+1) A_{1}\right)=k\left(A_{1}, A_{2}\right)
$$

in the distributional sense. Since $N$ is $C^{\infty}$ with bounded derivatives on bounded sets, $k$ is $C^{\infty}$ from $\Omega_{3}^{1}\left(S^{3}, \mathbf{g}\right) \oplus \Omega_{2}^{1}\left(S^{3}, \mathbf{g}\right)$ to $\Omega_{1}^{1}\left(S^{3}, \mathbf{g}\right)$, with bounded derivatives on bounded sets. It follows from Theorem 3 that the map $u \mapsto$ $X_{0} h(u)$ is $C^{\infty}$ from $U_{\pi}$ to $\mathbf{V}$.

For each $i,\left.X_{i} A\right|_{t=0}$ and $\left.X_{0} X_{i} A\right|_{t=0}$ are well-defined distributions by the theory of the Cauchy problem. Then for $1 \leq i \leq 3$ we have

$$
\left.\left(X_{i} A, X_{0} X_{i} A\right)\right|_{t=0}=\left(V_{i} B_{1}, V_{i} B_{2}\right),
$$

while for $i=0$ we have

$$
\left.\left(X_{0} A, X_{0}^{2} A\right)\right|_{t=0}=\left(B_{2}, B_{3}\right) .
$$

For each $i$ it is evident that the map $\left.u \mapsto\left(X_{i} A, X_{0} X_{i} A\right)\right|_{t=0}$ is $C^{\infty}$ from $\mathbf{H}$ to $\Omega_{1}^{1}\left(S^{3}, \mathbf{g}\right) \oplus \Omega_{0}^{1}\left(S^{3}, \mathbf{g}\right)$. Thus by Lemma 5 and the remarks following, we may define

$$
\left(X_{i} A\right) \mid C_{+}=T_{+}\left(X_{i} h(u),\left.\left(X_{i} A, X_{0} X_{i} A\right)\right|_{t=0}\right),
$$

and conclude that $u \mapsto\left(X_{i} A\right) \mid C_{+}$is a $C^{\infty}$ function from $U_{\pi}$ to $\mathbf{H}\left(C_{+}\right)$.

We use a similar argument for $\left(X_{0} X_{i} A\right) \mid C_{+}$. Given $u \in \mathbf{X}$ we have

$$
(\square+1) X_{0} X_{i} A=X_{0} X_{i} h(u)
$$

in the distributional sense. By (7) we have

$$
X_{0} X_{i} h(u)=X_{i} k\left(A_{1}, A_{2}\right) \text {. }
$$


For $1 \leq i \leq 3$, since $X_{i} k$ is $C^{\infty}$ from $\Omega_{3}^{1}\left(S^{3}, \mathbf{g}\right) \oplus \Omega_{2}^{1}\left(S^{3}, \mathbf{g}\right)$ to $\Omega_{0}^{1}\left(S^{3}, \mathbf{g}\right)$, it follows from Theorem 3 that the map $u \mapsto X_{0} X_{i} h(u)$ is $C^{\infty}$ from $U_{\pi}$ to $\mathbf{V}$. For $i=0$ we obtain the same conclusion as follows. By the chain rule we have

$$
X_{0}^{2} h(u)=X_{0} k\left(A_{1}, A_{2}\right)=k_{1}\left(A_{1}, A_{2}\right) A_{2}+k_{2}\left(A_{1}, A_{2}\right) A_{3}
$$

where $k_{i}$ denotes the Fréchet derivative with respect to $A_{i}$ of the expression for $k\left(A_{1}, A_{2}\right)$. It follows from the Sobolev inequalities that the right side is $C^{\infty}$ from $\mathbf{H}$ to $\Omega_{0}^{1}\left(S^{3}, \mathbf{g}\right)$, with bounded derivatives on bounded sets (see also Lemma 2.2 of [4]). Thus by Theorem 3, the map $u \mapsto X_{0}^{2} h(u)$ is $C^{\infty}$ from $U_{\pi}$ to $\mathrm{V}$.

For each $i,\left.X_{0} X_{i} A\right|_{t=0}$ and $\left.X_{0}^{2} X_{i} A\right|_{t=0}$ are well-defined distributions, by the theory of the Cauchy problem. For $1 \leq i \leq 3$ we have

$$
\left.\left(X_{0} X_{i} A, X_{0}^{2} X_{i} A\right)\right|_{t=0}=\left(V_{i} B_{2}, V_{i} B_{3}\right),
$$

and for $i=0$ we have

$$
\left.\left(X_{0}^{2} A, X_{0}^{3} A\right)\right|_{t=0}=\left(B_{3},-(\Delta+1) B_{2}+k\left(B_{1}, B_{2}\right)\right) .
$$

For each $i$, it is evident that the map $\left.u \mapsto\left(X_{0} X_{i} A, X_{0}^{2} X_{i} A\right)\right|_{t=0}$ is $C^{\infty}$ from $U_{\pi}$ to $\Omega_{1}^{1}\left(S^{3}, \mathbf{g}\right) \oplus \Omega_{0}^{1}\left(S^{3}, \mathbf{g}\right)$. Thus by Lemma 5 we may define

$$
\left(X_{0} X_{i} A\right) \mid C_{+}=T_{+}\left(X_{0} X_{i} h(u),\left.\left(X_{0} X_{i} A, X_{0}^{2} X_{i} A\right)\right|_{t=0}\right),
$$

and note that $u \mapsto\left(X_{0} X_{i} A\right) \mid C_{+}$is $C^{\infty}$ from $U_{\pi}$ to $\mathbf{H}\left(C_{+}\right)$.

By the above theorem there exist continuous "wave operators" $W_{ \pm}: \mathbf{X} \rightarrow$ $\mathbf{H}\left(C_{ \pm}\right)$such that $W_{ \pm}(u)=A \mid C_{ \pm}$where $A \in \Omega^{1}(\widetilde{\mathbf{M}}, \mathbf{g})$ is as in the theorem. The relation between these wave operators and the scattering operator $S$ is given as follows:

Theorem 6. Let $U: \mathbf{H}\left(C_{+}\right) \rightarrow \mathbf{H}\left(C_{-}\right)$be the isomorphism of real Hilbert spaces given by

$$
(U A)(t, x)=A(t+\pi,-x) \quad(t, x) \in C_{+} \subset \mathbf{R} \times S^{3} .
$$

Then we have $W_{-} S=U W_{+}$.

Proof. Suppose $u \in \mathbf{X}$, and let $A \in \Omega^{1}(\tilde{\mathbf{M}}, \mathbf{g})$ be the corresponding solution of the Yang-Mills equation in temporal gauge, as in the statement of Theorem 5. Then if $B \in \Omega^{1}(\widetilde{\mathbf{M}}, \mathbf{g})$ denotes the solution of the Yang-Mills equation in temporal gauge corresponding to $S u \in \mathbf{X}$, by the definition of $S$ we have $B(t, x)=A(t+\pi,-x)$. Thus

$$
W_{-} S u=B \mid C_{-}=U\left(A \mid C_{+}\right)=U W_{+} u .
$$

Acknowledgments. The author wishes to thank Irving Segal and Zheng-fang Zhou for many discussions concerning the Goursat problem and the Yang-Mills equations, without which this work could not have been done. 


\section{REFERENCES}

1. S. M. Paneitz and I. E. Segal, Analysis in space-time bundles. I, J. Funct. Anal. 47 (1982), 78-142.

2. Y. Choquet-Bruhat and D. Christodoulou, Existence of global solutions the Yang-Mills, Higgs, and spinor field equations in 3+1 dimensions, Ann. Ecole. Norm. Sup. (4) 14 (1981), 481-506.

3. D. Eardley and V. Moncrief, The global existence of Yang-Mills-Higgs fields on 4-dimensional Minkowski space, Comm. Math. Phys. 83 (1982), 121-191, 193-212.

4. Y. Choquet-Bruhat, S. M. Paneitz and I. E. Segal, The Yang-Mills equations on the universal cosmos, J. Funct. Anal. 53 (1983), 112-150.

5. I. E. Segal, Reduction of scattering to an invariant finite displacement in an ambient space-time, Proc. Nat. Acad. Sci. U.S.A. 81 (1984), 7266-7268.

6. J. Baez, I. E. Segal and Z. Zhou, The global Goursat problem and scattering for nonlinear wave equations (to appear).

7. J. Baez, Scattering and the geometry of the solution manifold of $\square f+\lambda f^{3}=0$, J. Funct. Anal. (to appear).

8. D. Bleecker, Gauge theory and variational principles, Addison-Wesley, Reading, Mass. 1981.

9. V. Moncrief, Reduction of the Yang-Mills equations, Differential Geometrical Methods in Mathematical Physics, Lecture Notes in Math., vol. 836, Springer, New York 1980.

10. I. E. Segal, Non-linear semi-groups, Ann. of Math. 78 (1964), 339-364.

11. __, Differential operators in the manifold of solutions of a non-linear differential equation, $\mathrm{J}$. Math. Pures Appl. 44 (1965), 71-132.

Department of Mathematics, Yale University, New Haven, Connecticut 06520 95121

Current address: Department of Mathematics, University of California, Riverside, California 